other cardiovascular disease, and noncancer respiratory disease.

Acknowledging that these results are restricted to patients with airway obstruction, the authors conclude that mortality can be reduced significantly by the introduction of intensive smoking-cessation programs.

Original article Anthonisen NR et al. (2005) The effects of a smoking cessation intervention on 14.5-year mortality. Ann Intern Med 142: 233-239

\section{Zinc deficiency increases esophageal cancer risk}

Animal studies have linked zinc deficiency to an increased risk of esophageal cancer, which is thought to be mediated by certain nitrosamines. This effect has been difficult to demonstrate in humans, however, because neither blood levels of zinc nor estimates of dietary zinc intake provide an accurate picture of the concentration of this mineral in the body's tissues. Abnet et al. have addressed this problem by measuring the concentration of zinc and other minerals directly in esophageal biopsy specimens, using X-ray fluorescence spectroscopy.

The samples were obtained from a population in Linzhou, China, where the diet contains large quantities of whole grain and little meat and, therefore, tends to be low in zinc. A total of 60 specimens were from subjects who subsequently developed esophageal squamouscell carcinoma during the 16-year Dysplasia Trial; 72 samples were from individuals who did not develop the disease. Baseline concentrations of zinc, copper, iron, nickel, and sulfur were measured by $\mathrm{X}$-ray fluorescence at two sites within each tissue sample.

During the 16-year follow-up, participants in the highest zinc quartile were at a significantly lower risk of developing esophageal cancer than those in the lowest quartile (hazard ratio $0.21,95 \% \mathrm{Cl} 0.065-0.680$ ). The corresponding disease-free survival rates were $90 \%$ and $65 \%$ for the two groups, respectively, and the association was statistically significant across quartiles $(P=0.015)$. Sulfur concentration also appeared to be inversely related to esophageal cancer risk when the highest and lowest quartiles were compared, but this association was not significant across quartiles. None of the other minerals tested showed any such relationship.
In summary, the study provides evidence for the role of zinc deficiency in the development of esophageal squamous-cell carcinoma in humans. The authors note that the approach described could be used to study other nutritional or toxic elements and their relationship with disease.

Original article Abnet CC et al. (2005) Zinc concentration in esophageal biopsy specimens measured by $\mathrm{X}$-ray fluorescence and esophageal cancer risk. J Natl Cancer Inst 97: 301-306

\section{Whole-body CT screening: is it cost-effective?}

Whole-body CT screening-generally based on unenhanced scanning of the heart, chest, abdomen, and pelvis-promises to save lives through the early detection of heart disease and various cancers. A recent paper published in Radiology, however, has revealed that this strategy might not be cost-effective.

Beinfeld et al. created a hypothetical cohort of half a million self-referred, asymptomatic, 50 -year-old men in the US. They then used a decision analysis model to compare the costs and effectiveness of onetime, whole-body CT screening with those of routine care (i.e. no screening). The analysis covered eight diseases: abdominal aortic aneurysm, coronary artery disease, and ovarian, pancreatic, lung, liver, kidney and colon cancer. The model predicted the results of the CT screening test based on the disease-specific sensitivity and specificity of the technique. Disease prevalence data from the Surveillance, Epidemiology, and End Results program were used to assign patient disease status.

Earlier disease detection by whole-body CT screening extended life expectancy by only 6 days, at an average additional cost of $\$ 2,513$ per patient. This equated to an incremental cost-effectiveness ratio of $\$ 151,000$ per life-year saved, relative to survival with no screening. This suggests that whole-body CT screening is less cost-effective than the majority of healthcare interventions in the US. About one-third of the total costs of screening were attributed to follow-up in patients who had false-positive results.

Beinfeld et al. acknowledge that costeffectiveness models are "a necessary simplification of reality" and that their analysis did not account for all diseases that might be detected 